\title{
A Facile and Generic Strategy to Synthesize Large-Scale Carbon Nanotubes
}

\author{
Yong Hu, ${ }^{1,2}$ Ting Mei, ${ }^{2}$ Libo Wang, ${ }^{2}$ and Haisheng Qian' \\ ${ }^{1}$ Zhejiang Key Laboratory for Reactive Chemistry on Solid Surfaces and Institute of Physical Chemistry, \\ Zhejiang Normal University, Jinhua 321004, China \\ ${ }^{2}$ School of Electrical and Electronic Engineering, Nanyang Technological University, Singapore 639798 \\ Correspondence should be addressed to Yong Hu, yonghu@zjnu.edu.cn \\ Received 24 September 2009; Accepted 14 January 2010 \\ Academic Editor: Raymond Whitby
}

Copyright () 2010 Yong Hu et al. This is an open access article distributed under the Creative Commons Attribution License, which permits unrestricted use, distribution, and reproduction in any medium, provided the original work is properly cited.

An easy method to prepare carbon nanotubes (CNTs) has been demonstrated using a two-step refluxing and calcination process. First, a readily available inorganic salt, $\mathrm{Ni}\left(\mathrm{NO}_{3}\right)_{2} \cdot 6 \mathrm{H}_{2} \mathrm{O}$, used as the catalyst precursor was dissolved in the high-boiling-point organic solvents (alcohols or polyhydric alcohol) by refluxing at $190^{\circ} \mathrm{C}$ for 3 hours. After refluxing, $\mathrm{NiO}$ nanoparticles obtained in the solution act as the catalyst, and the organic refluxing solvents are used as the carbon source for the growth of CNTs. Second, CNTs are prepared by calcining the refluxed solution at $800^{\circ} \mathrm{C}$ in an $\mathrm{N}_{2}$ atmosphere for 3 hours. Results show that CNT growth possibly originates from carbon rings, with the nanotube walls growing perpendicular to these rings and forming a closed tube at the end.

\section{Introduction}

Since their emergence, carbon nanotubes (CNTs) [1] have demonstrated their versatility in a variety of applications such as supportedcatalysts for hydrogenation reactions [2], fuel cells [3], field emission devices [4], and nanoelectronic devices [5], due to their unique mechanical, chemical, and electrochemical properties [6,7]. Consequently, demand for the consumption of CNT materials is huge, making desirable their fast, efficient, high yield and cost effective fabrication.

Previously, a number of techniques have been developed for fabricating CNTs, including electric arc-discharge [8], laser ablation [9], chemical vapor deposition (CVD) [10, 11], pyrolysis [12], plasma enhanced CVD (PECVD) [13], laser assisted CVD (LACVD) [14], two-stage fluid bed reactor [15], aerosol method [16], solvothermal method $[17,18]$, and high pressure CO disproportionation process (HiPCO) [19]. Recently, Tang et al. reported a novel catalytic combustion method of synthesizing CNTs in situ in high yield from polypropylene as a carbon source in the presence of organic-modified clay and a supported Ni catalyst [20]. Most of the methods originate from the idea of obtaining adequate active atomic carbon species or clusters from carbon sources and assembling them into CNTs on catalysts. Although great efforts have been made in the development of synthesis methods, their numerous steps and the adoption of expensive or nonrenewable materials still make the methods complicated. Thus we look for a simple, low-cost, and effective method for the synthesis of CNTs.

Herein, we report a simple and low-cost strategy of CNT synthesis that adopts a refluxing process with readily available inorganic salts $\mathrm{Ni}\left(\mathrm{NO}_{3}\right)_{2} \cdot 6 \mathrm{H}_{2} \mathrm{O}$ as the catalyst precursor, and high-boiling-point (BP) di(ethylene glycol) (DEG), glycerol and ethylene glycol (EG), as solvents and carbon source. After refluxing, $\mathrm{NiO}$ nanoparticles obtained in the solution act as the catalyst for the growth of CNTs. The final CNT products are successfully prepared by calcining the refluxed solution at $800^{\circ} \mathrm{C}$ in an $\mathrm{N}_{2}$ atmosphere for 3 hours. The whole process is kept simple and easy to control because there are not too many parameters required for monitoring.

\section{Experimental}

2.1. Synthesis of NiO Nanoparticles and CNTs. Chemical reagents purchased from Sigma-Aldrich were used in the experiments with no further purification. For a typical 


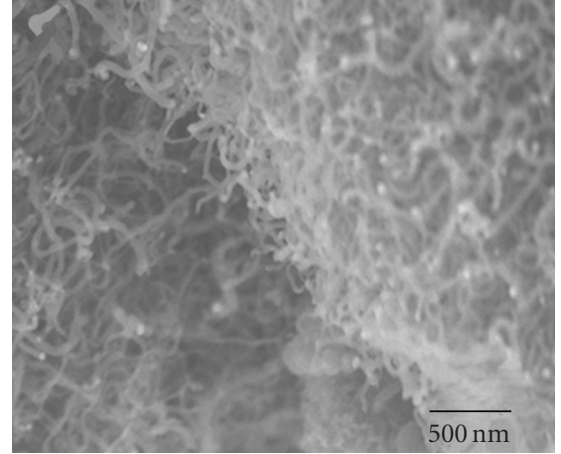

(a)

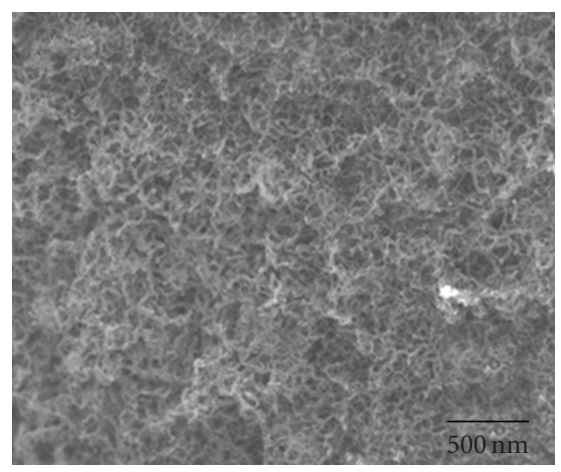

(c)

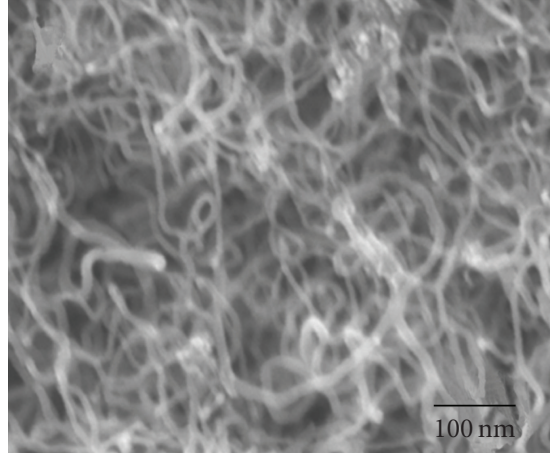

(b)

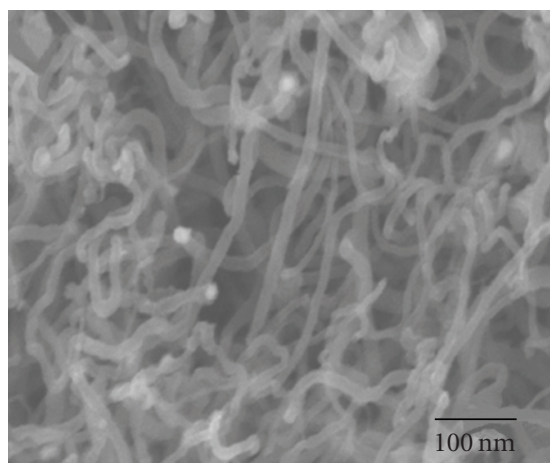

(d)

FIGURE 1: FE-SEM images of CNTs prepared by calcining DEG solution: (a) low magnification and (b) high magnification; FE-SEM images of CNTs prepared by calcining (c) glycerol solution and (d) EG solution.

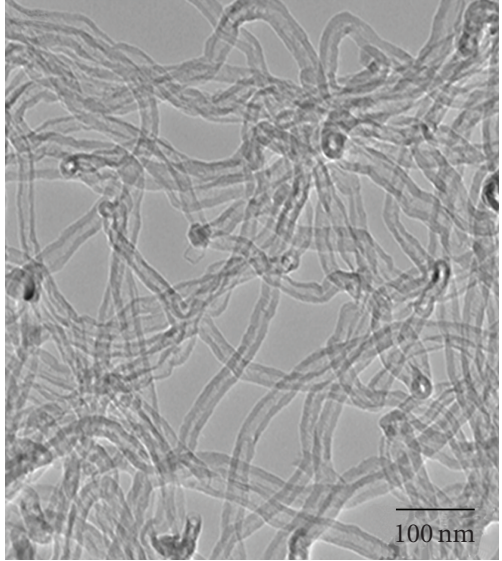

(a)

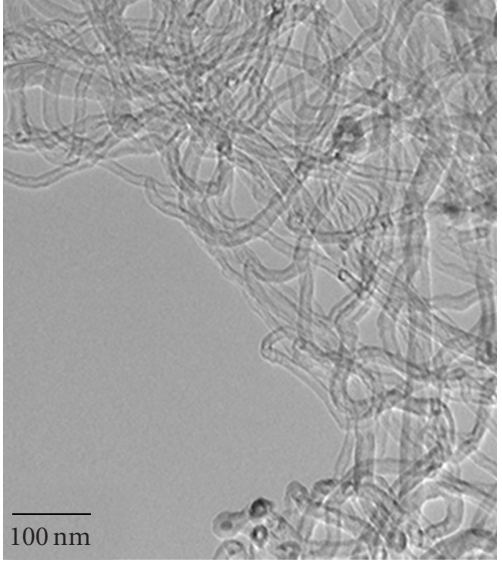

(b)

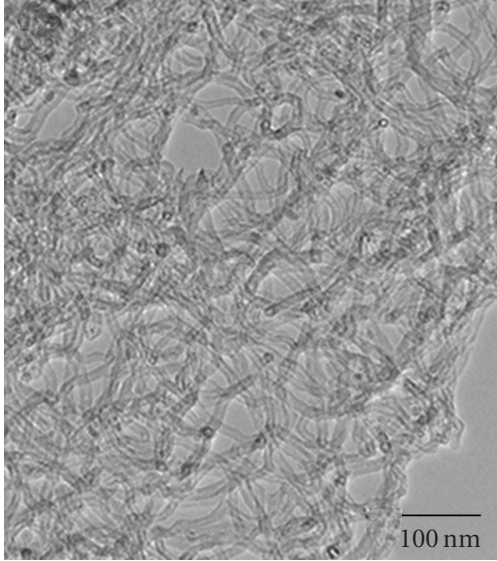

(c)

Figure 2: TEM images of CNTs prepared by calcining (a) DEG solution; (b) glycerol solution; (c) EG solution.

synthesis procedure, $0.0025 \mathrm{~mol}(0.73 \mathrm{~g})$ of the metal salt $\mathrm{Ni}\left(\mathrm{NO}_{3}\right)_{2} \cdot 6 \mathrm{H}_{2} \mathrm{O}$ was introduced and dissolved in $50 \mathrm{~mL}$ of DEG, glycerol, and EG solvent, in three round bottom flasks. The solutions were heated in an oil bath to form a clear solution under reflux with vigorous stirring. When the reflux temperature was increased to $190^{\circ} \mathrm{C}$, the clear solution then turned turbid. The reaction was maintained with stirring for aging for at least 3 hours at this fixed temperature.
The flasks were then cooled down to room temperature after the heat source was removed. Subsequently, the three refluxed solutions were injected into quartz crucibles with lid. The final CNT products were obtained by calcination at $800^{\circ} \mathrm{C}$ in an $\mathrm{N}_{2}$ atmosphere for 3 hours with a heating rate of $1^{\circ} \mathrm{C} \mathrm{min}^{-1}$. After calcination, the black precipitates were collected and washed with absolute ethanol, dilute $\mathrm{HCl}$ aqueous solution, and deionized water in sequence. Finally, 


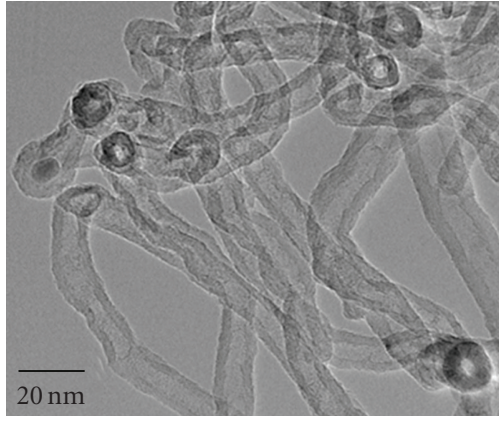

(a)

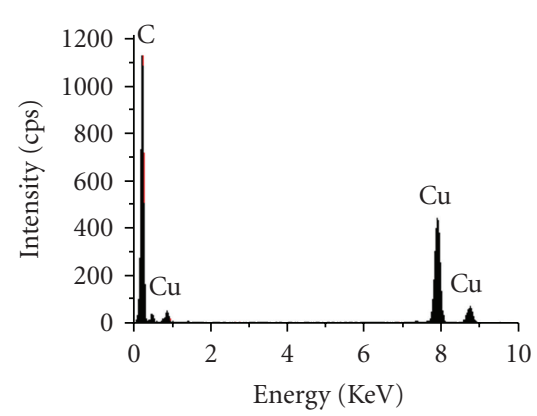

(d)

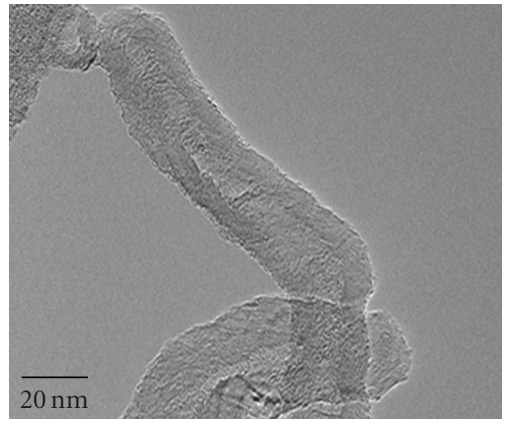

(b)

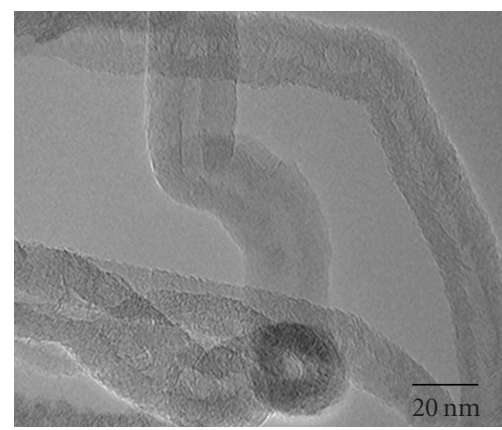

(e)

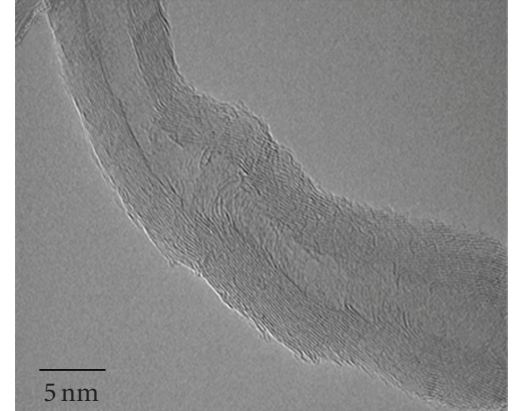

(c)

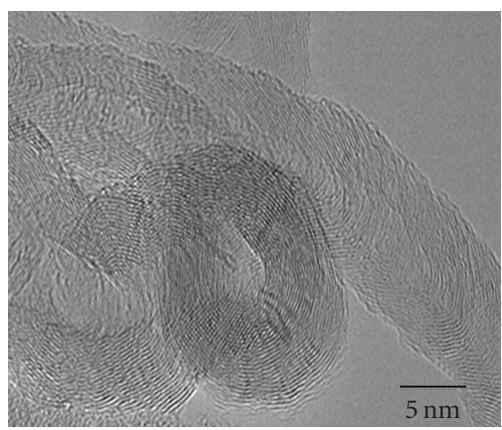

(f)

FIGURE 3: HRTEM images of CNTs: (a) nanotubes prepared by calcining DEG solution; (b) the closed bottom of a nanotube; (c) a section of nanotube wall; (d) the EDS pattern of a carbon ring; (e) the open tip of a carbon ring at low magnification and (f) at high magnification.

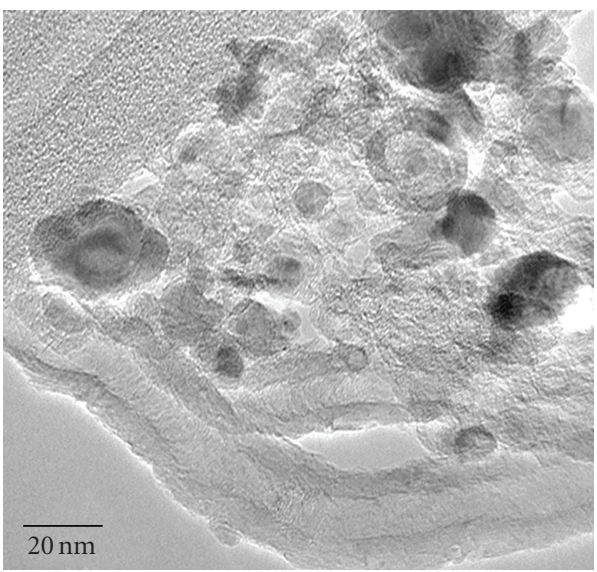

Figure 4: TEM image of CNTs prepared by calcination at $700^{\circ} \mathrm{C}$.

the obtained samples were dried in vacuum at $60^{\circ} \mathrm{C}$ for 6 hours.

2.2. Characterization. Morphological and structural examinations of the as-prepared products were performed using field-emission scanning electron microscopy (FE-SEM, JEOL JSM-6340F); transmission electron microscopy (TEM) and high resolution TEM (HRTEM) were conducted at $200 \mathrm{keV}$ with a JEM-2100F field emission machine, after dispersing the sample in ethanol and depositing several drops of the suspension on holey-carbon films supported by copper grids.
Energy-dispersive X-ray spectroscopy (EDS) was performed on a JEM-2100F TEM. The Raman spectrum was recorded at ambient temperature on a Witec Alpha 300 Raman spectrometer with an argon-ion laser at an excitation wavelength of $488 \mathrm{~nm}$.

\section{Results and Discussion}

The FE-SEM images of CNTs synthesized by calcining DEG solution are shown in Figure 1(a) (lowmagnification) and Figure 1(b) (highmagnification). Figures 1(c) and 1(d) show the images of the products prepared by calcining glycerol and EG solution. These images demonstrate that the approach presented in this paper offers a large-scale production yield for CNTs. These CNT samples present a uniform tube diameter $(\sim 15 \mathrm{~nm})$ and a tube length ranging from hundreds of nanometers to several microns.

The TEM images (Figure 2) reveal that the CNTs synthesized by this method are multiwall CNTs (MWCNTs) with strong graphitic structure. Figures 2(a), 2(b), and 2(c) show the characteristics of the MWCNTs with open tips as produced in all solutions. The nanotube sizes observed in these images are in good agreement with those observed using FE-SEM. A further investigation on the tubular structure of the HRTEM image shown in Figure 3(a) clearly reveals an open tip and a closed bottom, which validates that the growth of the nanotubes originated from carbon rings resulting in the formation of closed bottoms. Figures 3(b) and $3(\mathrm{c})$ show the closed bottom of nanotube and a section of 


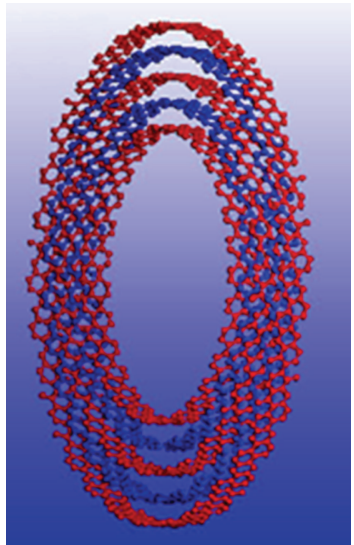

(a)

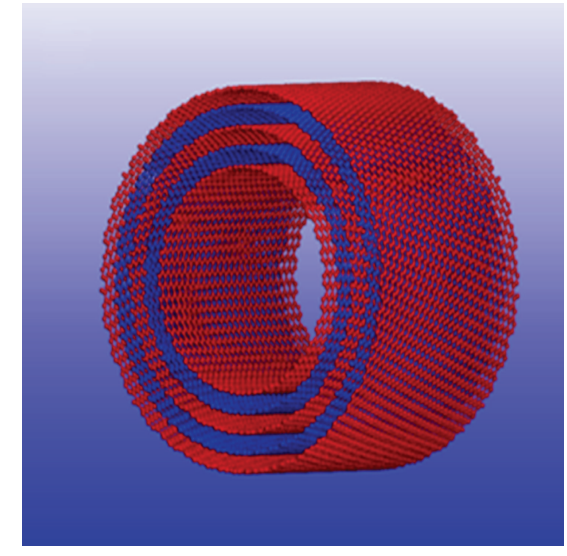

(b)

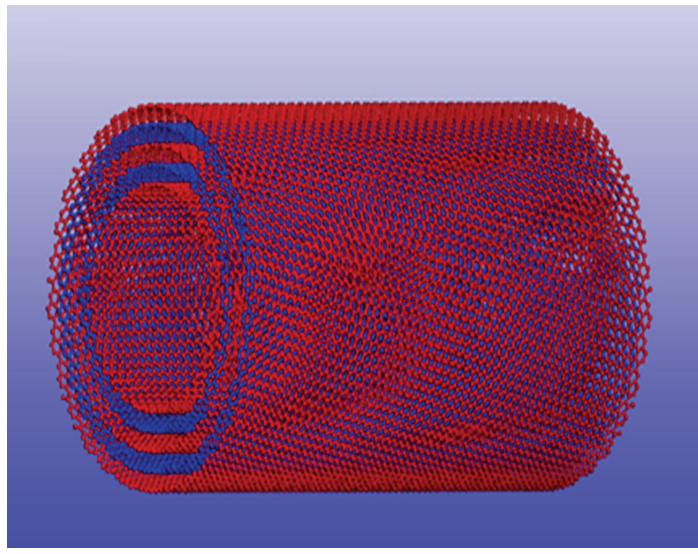

(c)

FIGURE 5: Schematic of CNT growth: (a) the formation of the original multi-layer carbon rings; (b) the growth of multi-layer nanotube walls grow perpendicular to the carbon rings; (c) the formed nanotube with an open tip and a closed bottom.

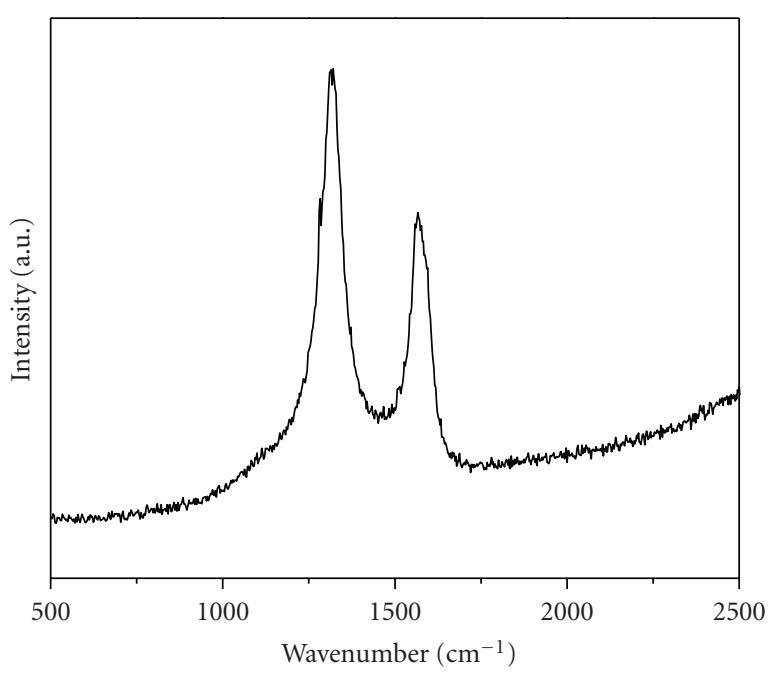

Figure 6: Raman spectrum of CNTs.

nanotube wall, which are clearly graphitized. The interlayer spacing in the multiwalls is about $0.34 \mathrm{~nm}$, corresponding to the lattice parameter of graphite carbon in the (002) plane [1]. EDS performed on a ring tip (Figure 3(d)) indicates that the tip only contains carbon; no other element was found. Figures 3(e) and 3(f) show the open tip of a carbon ring at low magnification and at high magnification.

In the refluxing process, $\mathrm{NiO}$ nanoparticles used as catalyst could be obtained via the pyrolysis of common metal salts $\left(\mathrm{Ni}\left(\mathrm{NO}_{3}\right)_{2} \cdot 6 \mathrm{H}_{2} \mathrm{O}\right)$ in a high $\mathrm{BP}$ organic solvent which acts as a carbon source in the next step. In the calcination process, nucleations of multilayer carbon rings were being formed under the catalyst effect of the $\mathrm{NiO}$ nanoparticles, and nanotubes were assembled along the ring axial growth. Meanwhile, because they were observed products at $700^{\circ} \mathrm{C}$ (Figure 4), we believe that the great amount of ring-like products further favored the growth of nanotubes from the multi-layer carbon rings. In addition, for MWCNTs, it is quite likely that the presence of the outer wall stabilizes the inner wall, keeping it open for continuous growth [21]. A possible CNT growth mechanism was depicted in detail in Figure 5.

The representative Raman spectrum (Figure 6) of the product calcined in refluxing DEG solution at $800^{\circ} \mathrm{C}$ shows the typical features of MWCNTs [1]. The spectrum exhibits two peaks at 1318 and $1570 \mathrm{~cm}^{-1}$, indicating the graphite structure of the nanotubes. According to the analysis of Kasuya et al. [22], the complex structure as characterized by the $1540-1600 \mathrm{~cm}^{-1}$ region can be understood by zonefolding of the graphite phonon dispersion relation. The IR spectrum demonstrates the peak frequencies of the graphite (G) mode at $1570 \mathrm{~cm}^{-1}$ and contains disorder modes at $1318 \mathrm{~cm}^{-1}$ (D).

\section{Conclusions}

We have successfully synthesized CNTs of uniform diameter on a large scale through a novel refluxing and calcination solution process, in which reflux solvents were used as the carbon source and $\mathrm{NiO}$ nanoparticles obtained after refluxing were used as the catalyst. The products prepared in this approach were no toxic and corrosive reagents. This approach allows adoption of other metal salts and high BP organic solvents for further exploration. Due to merits such as its simplicity, low cost, high purity, good controllability, and high yield, we believe that this method can be exploited at the scale of industrial production.

\section{Acknowledgments}

This material is based on research supported by the Scientific Research Foundation for the Returned Overseas Chinese Scholars, State Education Ministry, and the Singapore National Research Foundation under CRP Award no. NRFG-CRP 2007-01. 


\section{References}

[1] S. Iijima, "Helical microtubules of graphitic carbon," Nature, vol. 354, no. 6348, pp. 56-58, 1991.

[2] C. Pham-Huu, N. Keller, L. J. Charbonniere, R. Ziessel, and M. J. Ledoux, "Carbon nanofiber supported palladium catalyst for liquid-phase reactions. An active and selective catalyst for hydrogenation of $\mathrm{C}=\mathrm{C}$ bonds," Chemical Communications, no. 19, pp. 1871-1872, 2000.

[3] C. Wang, M. Waje, X. Wang, J. M. Tang, R. C. Haddon, and Y. Yan, "Proton exchange membrane fuel cells with carbon nanotube based electrodes," Nano Letters, vol. 4, no. 2, pp. 345-348, 2004.

[4] S. Fan, M. G. Chapline, N. R. Franklin, T. W. Tombler, A. M. Cassell, and H. Dai, "Self-oriented regular arrays of carbon nanotubes and their field emission properties," Science, vol. 283, no. 5401, pp. 512-514, 1999.

[5] P. J. de Pablo, E. Graugnard, B. Walsh, R. P. Andres, S. Datta, and R. Reifenberger, "A simple, reliable technique for making electrical contact to multiwalled carbon nanotubes," Applied Physics Letters, vol. 74, no. 2, pp. 323-325, 1999.

[6] R. H. Baughman, A. A. Zakhidov, and W. A. de Heer, "Carbon nanotubes-the route toward applications," Science, vol. 297, no. 5582, pp. 787-792, 2002.

[7] J.-M. Nhut, L. Pesant, J.-P. Tessonnier, et al., "Mesoporous carbon nanotubes for use as support in catalysis and as nanosized reactors for one-dimensional inorganic material synthesis," Applied Catalysis A, vol. 254, no. 2, pp. 345-363, 2003.

[8] T. W. Ebbesen and P. M. Ajayan, "Large-scale synthesis of carbon nanotubes," Nature, vol. 358, no. 6383, pp. 220-222, 1992.

[9] A. Thess, R. Lee, P. Nikolaev, et al., "Crystalline ropes of metallic carbon nanotubes," Science, vol. 273, no. 5274, pp. 483-487, 1996.

[10] D. Y. Kim, C.-M. Yang, Y. S. Park, et al., "Characterization of thin multi-walled carbon nanotubes synthesized by catalytic chemical vapor deposition," Chemical Physics Letters, vol. 413, no. 1-3, pp. 135-141, 2005.

[11] G.-Y. Xiong, D. Z. Wang, and Z. F. Ren, "Aligned millimeterlong carbon nanotube arrays grown on single crystal magnesia," Carbon, vol. 44, no. 5, pp. 969-973, 2006.

[12] A. Govindaraj and C. N. R. Rao, "Organometallic precursor route to carbon nanotubes," Pure and Applied Chemistry, vol. 74, no. 9, pp. 1571-1580, 2002.

[13] K. H. Jung, J.-H. Boo, and B. Hong, "Synthesis of carbon nanotubes grown by hot filament plasma-enhanced chemical vapor deposition method," Diamond and Related Materials, vol. 13, no. 2, pp. 299-304, 2004.

[14] S. N. Bondi, W. J. Lackey, R. W. Johnson, X. Wang, and Z. L. Wang, "Laser assisted chemical vapor deposition synthesis of carbon nanotubes and their characterization," Carbon, vol. 44, no. 8, pp. 1393-1403, 2006.

[15] W. Qian, T. Liu, F. Wei, Z. Wang, and Y. Li, "Enhanced production of carbon nanotubes: combination of catalyst reduction and methane decomposition," Applied Catalysis A, vol. 258, no. 1, pp. 121-124, 2004.

[16] A. G. Nasibulin, A. Moisala, H. Jiang, and E. I. Kauppinen, "Carbon nanotube synthesis from alcohols by a novel aerosol method," Journal of Nanoparticle Research, vol. 8, no. 3-4, pp. 465-475, 2006.
[17] L. Jiang and L. Gao, "Carbon nanotubes-magnetite nanocomposites from solvothermal processes: formation, characterization, and enhanced electrical properties," Chemistry of Materials, vol. 15, no. 14, pp. 2848-2853, 2003.

[18] Y. Jiang, Y. Wu, S. Zhang, et al., "A catalytic-assembly solvothermal route to multiwall carbon nanotubes at a moderate temperature," Journal of the American Chemical Society, vol. 122, no. 49, pp. 12383-12384, 2000.

[19] P. Nikolaev, M. J. Bronikowski, R. K. Bradley, et al., "Gasphase catalytic growth of single-walled carbon nanotubes from carbon monoxide," Chemical Physics Letters, vol. 313, no. 1-2, pp. 91-97, 1999.

[20] T. Tang, X. Chen, X. Meng, H. Chen, and Y. Ding, "Synthesis of multiwalled carbon nanotubes by catalytic combustion of polypropylene," Angewandte Chemie International Edition, vol. 44, no. 10, pp. 1517-1520, 2005.

[21] T. Guo, P. Nikolaev, A. G. Rinzler, D. Tománek, D. T. Colbert, and R. E. Smalley, "Self-assembly of tubular fullerenes," Journal of Physical Chemistry, vol. 99, no. 27, pp. 10694-10697, 1995.

[22] A. Kasuya, Y. Sasaki, Y. Saito, K. Tohji, and Y. Nishina, "Evidence for size-dependent discrete dispersions in singlewall nanotubes," Physical Review Letters, vol. 78, no. 23, pp. 4434-4437, 1997. 

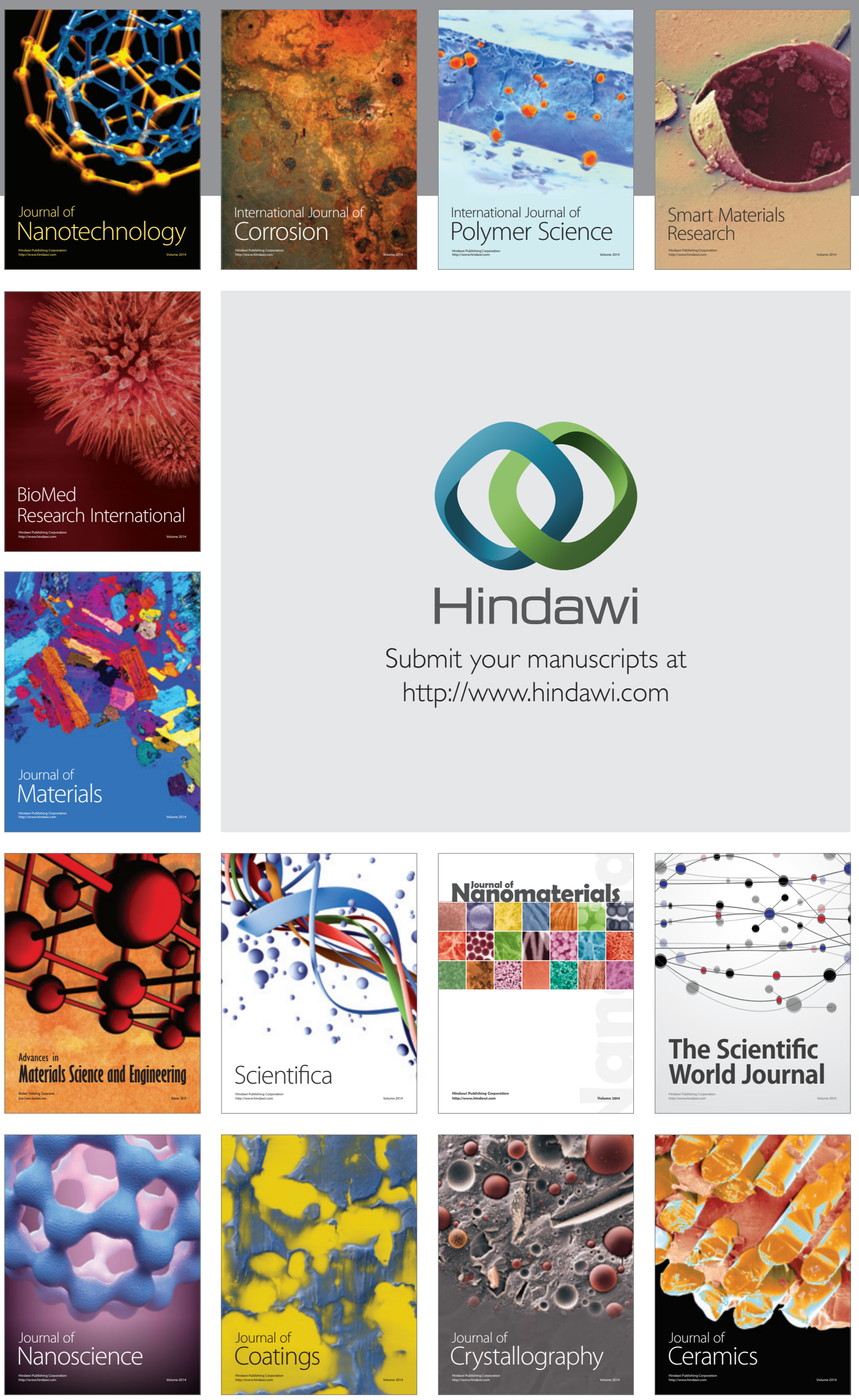

The Scientific World Journal

Submit your manuscripts at

http://www.hindawi.com

\section{World Journal}

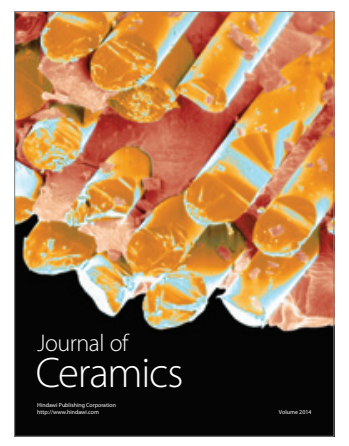

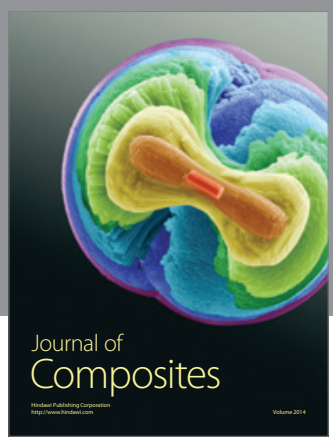
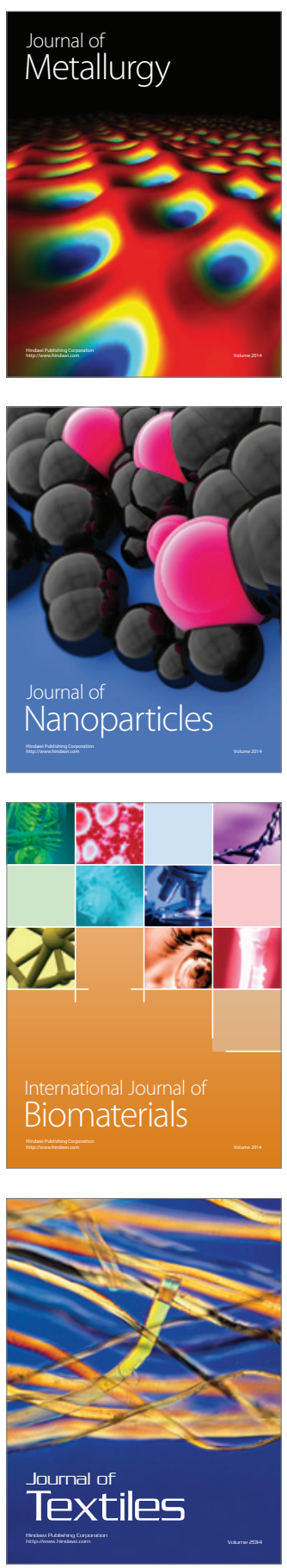